\title{
INNOVATIONS IN THE GRAPHIC ARTS, MEDIA AND PACKAGING FIELDS
}

\author{
Anastasios E. Politis \\ HELGRAMED - the Hellenic Union of Graphic Arts and \\ Media Technology Engineers, Athens, Greece \\ University of West Attica, Egaleo, Greece \\ Hellenic Open University, Patras, Greece
}

\begin{abstract}
In recent years, some have predicted that printing and graphic arts will be eliminated by electronic and digital media. Within these predictions there was no future physical carriers of communication and paper would be replaced by monitors and screens, tablets and mobile devices.

Back at DRUPA 1982, the introduction of the so-called "Desktop Publishing" has led to an exhibition under the title "Goodbye Gutenberg", predicting also that print was under threat by new electronic technologies. Hence, until today, graphic arts industry, printing and publishing and most of all packaging, present a continuous positive development, together with structural changes in the entire spectrum of research, industry and education.

Graphic Arts today is characterized by rapid technological developments, restructuring of design, management and production processes, and the application of various innovations. One of the most important indicators is the integration of basic and applied research in many fields, which leads to a new shape of the sector. In the present paper, issues concerning the following will be addressed (among others):

- Typography and its importance in the digital media domain

- Evolution, Innovations and main trends in the graphic arts, printing and packaging industry

- Digitalization and printing production (Industry 4.0, 3D printing)

- The concept of Industrial Printing
\end{abstract}

In this keynote paper, effort will be given to illustrate the trends and developments in graphic arts fields. Further, the parameters confirming that printing, paper, typography, bookbinding and packaging will stay alive in the digital era will be analyzed. In addition, the graphic arts sector and its positioning in the so-called "post-industrial" society, will be investigated and the new directions for the graphic arts research, industry and business as well as the requirements for education will be identified.

Key words: graphic arts innovations, typography, printing, packaging, industry 4.0, industrial printing

\section{IS PRINT DEAD?}

Many have predicted that printing and in general, the graphic arts sector will disappear... Those following the evolution and developments of the sector in the last decades might remember an exhibition during DRUPA 1982, with the title: "GOOD BYE GUTENBERG". In addition, some other predictions stated that print will be replaced by digital media. Well, it seems that up to today, such statements and predictions on the "death" of print do not seem to be a reality. Our field and the related sectors show a rather stable condition in terms of turnover and production, at least in Europe and North America, whereas in other continents, such as Asia, print is booming. The main driver for this is the packaging sector, which seems to be the most significant stakeholder of the printing industry worldwide. To give an example, even in Greece with the continuous crisis that takes place the last decade and where some 30\% of printing companies disappeared, packaging printing shows a stable turnover increase of some 3,5\%.

Of course, nowadays, we are witnessing the expansion of digital communication and in particular the use of internet, social media, the enormous expanding of mobile devices, apps, and the web. However, the characteristics and in particular the nature of printed substrates combined with advanced technologies, such as finishing technologies reveal the strength of printing world and enhance the added value for a better communication. Haptic communication is the principal driver for this enhancement.

\subsection{Print is NOT dead! - Some key paradigms}

Tim Freeman, President at Printing Industries Alliance in a report entitled "PRINT - The reports of my death have been greatly exaggerated!", states that "Like Mark Twain in the late 1800's, much has been 
written, fortunately prematurely, about the untimely demise of PRINT. As the world continues to examine the phenomenon of "fake news", data hacks, and advertising ROI, the Print Drives America Foundation continues to promote the concept that PRINT is not only the largest communications media of all (larger than broadcast, internet, new media and all other media combined) but most importantly, the most effective" (Pialliance, 2018). In an article written by Morag published in "The Drum", a global media platform and the biggest marketing website in Europe, "print, is re-affirming the reliability and authority of printed communication in so many ways" (Blazey, 2018). Following this, Morag states that "perhaps we should all stop proclaiming that certain media are 'dead' or 'alive'. Undeniably both circulations of and ad spend in print media have fallen over recent years, but the fact is that print should still be considered a viable choice of medium depending on the end purpose of the communications.

Further, the article presents the Facebook's newspaper apology ad (Figure 1). The very gravity of Facebook's situation dictated a print apology. Print is where the Facebook story first broke, and so it is appropriate that the apology starts here. There is also the question of context: Print is a news environment, not a social environment - the connotations of apologising via social may have undermined the apology. It's no surprise that Facebook ran its apology in print - the combination of heritage, authority, gravity, context, performance and audience make it the most suitable medium for "comms" like this (Blazey, 2018).

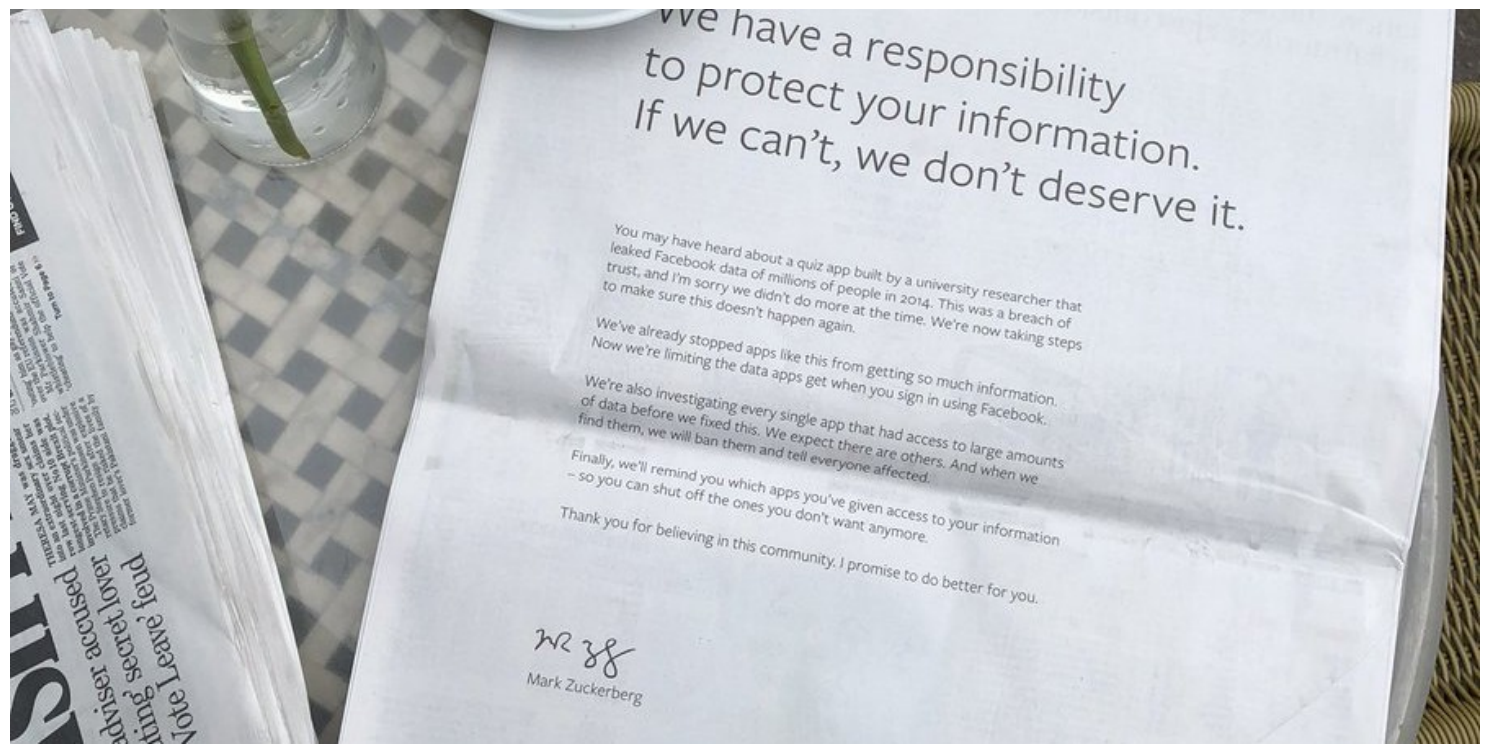

Figure 1: Facebook's newspaper apology ad

Source: http://www.thedrum.com/opinion/2018/03/29/print-dead-then-why-do-even-the-tech-giants-use-it-theirapology-ads

Another view on print media in relation with internet is stated at YouGov platform. "In a world of fake news, print is more trusted than Online media, particularly at the local level. $74 \%$ of respondents agree that they trusted the news and information in their local newspaper" over online sources. This trend is replicated by journalists - as published in WARC, Ogilvy Media Influence's survey of 255 media professionals found that " $52 \%$ of (journalists) now believe traditional media to be the most trusted source of news while social media is seen as the biggest contributor to the growth of fake news" (Blazey, 2018). As a conclusion, "Print possesses gravity and authority not held by newer channels. It has historically been the domain of births, deaths, marriages and major announcements As such, this sense of authority has always had applications for marketing - brand apologies and even product recalls traditionally run in print".

\section{TYPOGRAPHY AND ITS IMPORTANCE IN THE DIGITAL MEDIA DOMAIN}

This leads us to consider the role of Typography in the digital age. Whereas typographic printing can be found only in collections and printing museums, the very role and significance of Typography is arising. This is taking place mainly because the principles of Typography are those that define quality and guidelines in every form of media, no matter the technology used (Politis, 2017). 
Typography, together with paper and print, is directly related with quality reading. In addition, the haptic performance and sense of nature, is absolutely positively combined with humans, through handwriting. A study conducted by Vincent (Vincent, 2016), examined the topics of both reading and writing in the educational setting of universities.

The study revealed that using pen and paper for writing and reading as well as, and combined with, digital technologies remains part of the normative practices of University students. Motivations for using paper and pen are influenced by the haptic qualities of reading and writing - the feel and the smell of the paper and the grasp of the pen, the turn of the page, and extend also to the practical usefulness of note taking and writing in margins while reading.

There is no doubt that students have embraced the use of digital technologies in the educational setting of their university with enthusiasm but they have also found that the affordances of chirographic writing and the use of paper have special qualities that cannot be matched by digital media (Vincent, 2016). As such, the normative practices of students show that there is still a demand for pen and paper as well as keyboard and screen and that in some instances the use of paper is preferred.

\section{DIGITAL TRANSFORMATION AND ITS IMPLICATIONS FOR GRAPHIC ARTS}

Digital technologies play today a quite significant role in the media and graphic arts domain. However, digital transformation, digitalization or digitization has various interpretations and different meanings, which can be shortly defined as follows (Politis, 2018):

- Digital transformation - digitization in production processes in the printing industry (e.g. digital printing, digital prepress)

- Digital transformation - digitization in media with the evolution of digital and mobile media

- Digital transformation - digitization in production operation, business models and management (e.g. Industry 4.0, 3D printing, additive manufacturing)

This rather arbitrary classification, helps us to clarify both positive and possibly negative implications of digitization in the graphic arts fields. The conclusion from this interpretation of digitization is that no matter the digital evolution, print is evolving as well, mostly positively. Industry 4.0 is the most prominent step towards a new era for printing in the digital domain (Politis, 2018).

\subsection{Industry 4.0 and graphic arts}

Industry 4.0 is described as the 4th industrial revolution. Industrial revolution is an old term, used principally to classify the various steps of evolution in industrial operations and processes. However, the concept of Industry 4.0 appears as a global trend regarding the evolution in industrial manufacturing in the years to come. As such, it seems as a necessity for all industrial and manufacturing sectors to take under consideration the evolution that this concept brings. Hence, the term consists of several elements, which need to be carefully addressed and analysed. This analysis should lead to the determination of potential benefits for manufacturing operations and processes in various sectors (Drexler, 2018). In figure 2, the four industrial revolutions are illustrated.

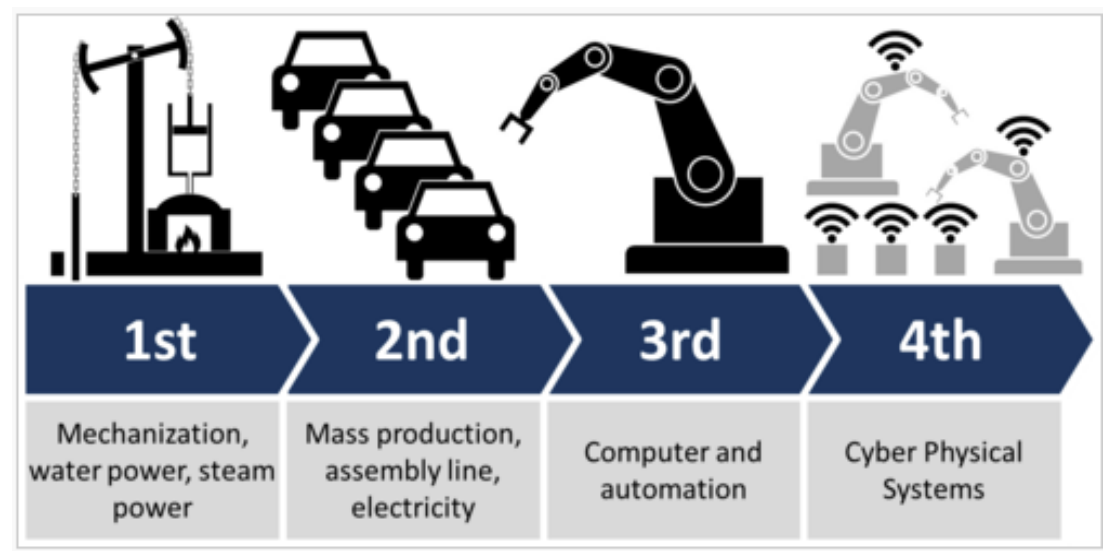

Figure 2: The four Industrial revolutions Source: https://en.wikipedia.org/wiki/Industry_4.0 
Industry 4.0 has been defined as a name for the current trend of automation and data exchange in manufacturing technologies. It includes cyber-physical systems, the Internet of things, cloud and cognitive computing and creates what has been called a "smart factory". Industry 4.0 appears as an interesting global trend regarding the evolution in industrial manufacturing in the years to come. Since it has various definitions, interpretations, and elements, there is a necessity for specific definition of the general concept into certain sectors. Digitisation is considered as a principal element of Industry 4.0 (Politis, 2018).

As it concerns the graphic/media, printing and packaging industries, the generic Industry 4.0 trend is applied with the combination of certain elements that fit better to the nature of processes and operations in the industry. Vendors, organizations and scientists from the graphic arts and media printing sectors could not stay out of the debate for manufacturing evolution caused by the Industry 4.0 trend. Numerous manufacturers are taking position within Industry 4.0. As such Industry 4.0 is classified / translated as Print 4.0, Finishing 4.0, Packaging 4.0 and Paper 4.0 (Politis, 2017).

A clear message from the investigation in the graphic/media sector is that not all Industry 4.0 elements are applicable at all sectors. The investigation for the graphic arts and related industries (printing, packaging, paper and finishing) show that generic elements of Industry 4.0 are adapted into specific applications in a more concrete manner at the level of manufacturers of graphic arts equipment and systems (Politis, 2017).

\subsection{Digital business models}

Extending digitization in the graphic arts and printing industry, Heidelberg claims that Digital business models are the future. (Heidelberger, 2018). According to Heidelberg, new digital business models such as software subscription contracts focus on the possibilities of cost-efficient print shop industrialization as digitization continues apace. Under the theme "Heidelberg goes digital, the new subscription business model offers a cost effective solution and simplified approach towards enabling end-to-end workflow automation and achieving optimal productivity, and a print shop's business success is increasingly determined by how efficiently it handles data (Heidelberger, 2018).

Further, as it is stated by Bohan, printing companies today have to navigate the requirements of shorter run lengths, faster turnaround times and increasing costs, all while facing relatively stable print prices and higher consumables prices. With all of these challenges, it's virtually impossible to maintain, yet alone increase, profitability without making changes to your current business model or operations (Bohan, 2018). Printing companies today have to navigate the requirements of shorter run lengths, faster turnaround times and increasing costs, all while facing relatively stable print prices and higher consumables prices. With all of these challenges, it's virtually impossible to maintain, yet alone increase, profitability without making changes to your current business model or operations. According to Bohan, "the key to profitability and the solution to current challenges may be no further than workflow. An advanced workflow, should consider whether or not it drives positive impact on six key areas of printing business:

- Enhancing Customer Interaction

- Reducing Touchpoints

- Driving Productivity and Uptime

- $\quad$ Reducing Waste and Inventory

- Optimizing Consistency and Repeatability

- $\quad$ Providing Business Intelligence

\section{CURRENT AND FUTURE TRENDS FOR GRAPHIC ARTS AND PRINTING INDUSTRY}

As it is presented at DRUPA 2020 advertisement, six principal fields will dominate the exhibition in 2020, namely Print, Packaging production, Functional printing, 3D Printing, Future technologies and Industrial printing (Drupa, 2018). According to DRUPA website, Industrial Printing means Automation, robotics and new workflows in the production process as the next generation of Industry 4.0 (Drupa, 2018). 


\subsection{Industrial printing}

Hence another interpretation for Industrial printing seems to be innovative concepts for a new era of printing, beyond traditional materials and substrates for totally new fields. According to this interpretation, Industrial printing is defined as the concept for applying print in medical and healthcare fields, in ceramics, and artificial "skin", photovoltaics, and decoration items and artifacts. Based on a study published by the Greek portal "graphicarts.gr", industrial printing expands the traditional printing processes in areas such as, Decoration, Multilayer materials, Ceramics, Fabrics and Glass (Graphicarts, 2018). According to the article, "Printing industry owners have to adapt to the evolving needs of consumers and buyers, which has allowed significant growth in four key market segments, namely decoration and multilayer materials, Printed electronics, Digital textile printing and 3D Printing. Smithers Pira's report - The Future of Operational and Industrial Printing by 2022 - estimates the value of the industrial and functional printing market at $\$ 76.9$ billion in 2017, predicting it will grow to $\$ 114.8$ billion by 2022 (Graphicarts, 2018).

\section{PACKAGING- DEVELOPMENTS AND TRENDS}

Packaging plays a crucial role in the world business and economy and it is extremely important for the global society and economy. Innovative developments at all levels are a mainstream procedure for packaging (supply chain, products and sales, transport, security, design, consumption). Packaging is nowadays, a multi-disciplinary field, a rapidly evolving science and a dynamic industry with continuous positive indicators. In addition, packaging is the principal stakeholder for printing, with some 55\% of printing worldwide to be directed on packaging Substrates (Politis, 2014).

A comprehensive research by Smithers Pira in its report on the Future of Global Packaging to 2022, shows that packaging demand will grow steadily at 2.9\% to reach $\$ 980$ billion in 2022 (Smitherspira et al, 2018). In addition, according to Marketsandmarkets, the global packaging printing market was valued at USD 328.95 Billion in 2015 and is projected to reach USD 574.47 Billion by 2026, at a CAGR of 5.3\% (Marketsandmarkets, 2018).All these illustrate the importance of packaging for the printing and graphic arts industry.

\subsection{Packaging and the environment}

The environment and its protection, the conservation of natural resources and energy saving, are nowadays key priorities for all modern societies. One of the most important features of packaging is its relationship to sustainability and environmental protection. The identification of practices, applications and procedures for controlling environmental and sustainability data relates to materials, production, handling and, more generally, to the lifecycle of the packaging.

Packaging, as an indispensable part of the product, but which is not consumed, is the most appropriate field where such policies (sustainability and environmental protection) are fully applicable (Pialliance, 2018). In this context, a variety of strategies and initiatives, as well as technologies, techniques and systems are being developed in the wider field of sustainability (Sarigiannidis et al, 2018). The environmental aspect of packaging is quite important since sustainability and the environment is considered as one of the principal issues for packaging production. Nowadays, packaging holistic design, materials and production implement sustainability concepts, in order to use less resources, recycle and protect the environment within the lifecycle of a product (Sarigiannidis, 2018).

\section{6. (SOME) CONCLUSIONS}

Printing and the graphic arts industry, show a rather positive development. Digitalization and digital transformation, together with innovations at all fields of the sector are the drivers towards this positive development. We, as researchers in the graphic arts fields should not only follow the trends, but lead the way on the transformation of our industry with intensive research for a bright future of our industry. This of course includes education as an integral part of the holistic evolution of graphic arts. 
Such new - innovative tasks may include printing processes, substrates and materials, the formation of new structures in the Industry (e.g. Cultural and Creative Industries), and the role of Graphic Arts. Some indicative advanced research topics are presented here, as they are derived from the research department of HELGRAMED - The Hellenic Union of Graphic Arts and Media Technology Engineers. They are presented as indicators and good practices for further research:

- Colour Management, Measurement and Certification

(Colour science - colour management systems)

- Holistic packaging design

- Innovative printing substrates and materials, inks and coatings

- $\quad$ Printing Science Hybrid printing, security and 3D printing

- Industrial printing

- Sustainability, environmental protection and energy efficiency

- Modern typographic design - Information and interface design

- Printed electronics and interactive information carriers - RFID-NFC

- Innovative business models, Life cycle Management, Lean Manufacturing, Production workflow management

- Industry 4.0 in the printing industry

- Standardization - A continuous development in ISO and other standards (e.g. ISO-12647)

\section{REFERENCES}

[1] Blazey, M.: "Print is dead' - then why do even the tech giants use it for their apology ads?", Thedrum, URL: http://www.thedrum.com/opinion/2018/03/29/print-dead-then-why-do-even-thetech-giants-use-it-their-apology-ads (last request: 2018-09-23).

[2] Bohan, M.: "How Prinect Production Manager Will Reshape Your Business", Heidelbergusa, URL: https://news.heidelbergusa.com/2017/11/prinect-production-manager-reshape/ (last request: 2018-09-18).

[3] Drexler, S.: "The 5 Factors of Industry 4.0, On digitizing Industry and Infrastructure, Industrial IOT/Industrie 4.0 Viewpoints", Industrial-iot, URL: https://industrial-iot.com/2016/08/5-factorsindustry-4-0/, (last request: 2018-10-11).

[4] Drupa exhibition 2020, URL: https://www.drupa.com/?utm_source=drupa20\%20besucher\&amp;utm_medium=email\&amp;utm _content=header\&amp;utm_campaign=news\%20okt18\%20en (2018-10-20).

[5] Graphicarts, The top 4 sectors are growing faster in industrial printing, Graphicarts, URL: http://www.graphicarts.gr/portal/showitem.php?artlid=8188 (2018-09-11).

[6] Heidelberger Druckmaschinen, "Digital business models are the future: Heidelberg achieves 100 worldwide installations of Prinect Production Manager", Heidelbergusa, URL: https://news.heidelbergusa.com/2018/10/digital-prinect-production-manager/ (last request: 2018-09-05).

[7] Markets and markets, Packaging Printing Market by Printing Ink, Marketsandmarkets, URL: https://www.marketsandmarkets.com/Market-Reports/packaging-printing-market153207109.html?gclid=CjwKCAjw8ajcBRBSEiwAsSky_ecQnTHgr4mN972nMDuCrB1C2b4npfF9cDby3 B1AvlbF4UwiwTxc_BoC_scQAvD_BwE (2018-10-13).

[8] Pialliance, Print Drives America Foundation, Pialliance, URL: http://pialliance.org/print-drivesamerica-foundation/, (last request: 2018-10-07).

[9] Politis, A.: "Industry 4.0: What does it mean for the Graphic Arts Industry?", The 45 ${ }^{\text {th }}$ IARIGAI Conference, (Warsaw, Poland, 2018), 4-7.

[10] Politis, A.: "The Graphic-Media Communication Science - Fields, Trends and the Contribution of Research Organizations", The Scientific conference - Evolution Innovation in Graphic-media Science: Research, Industry Innovation and the Role of Education, (Hellenic Union of Graphic Arts and Media Technology Engineers - HELGRAMED, Athens, Greece, 2017).

[11] Politis, A.: "Typography revisited: The importance of Typography in modern visual communication". Aepm annual conference 2017, (The Museum of Typography, Chania Crete, Greece, 2017).

[12] Politis, A: "Transforming packaging buyer requirements to efficient solutions for packaging production", ESKOWORLD Conference, 2014, (Athens, Greece, 2014). 
[13] Sarigiannidis Ch., Politis, A., Voutsinas, V.: "Packaging and Environment - a Comparative Analysis of MFCA, Full Costing and ABC Systems in Environmental Management Accounting (EMA)", The $1^{\text {st }}$ Hellenic Conference on Graphic Arts, (University of Ioannina, Ioannina, Greece, 2018).

[14] Smithers Pira, The Future of Global Packaging to 2022, Smitherspira, URL: https://www.smitherspira.com/industry-market-reports/packaging/the-future-of-global-packagingto-2022 (2018-10-19).

[15] Vincent, J.: "Students' use of paper and pen versus digital media in university environments for writing and reading - a cross-cultural exploration", Journal of Print Media and Media Technology Research, 5 (2), 97-106, 2016. doi: 10.14622/JPMTR-1602

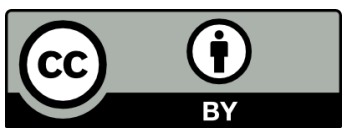

(C) 2018 Authors. Published by the University of Novi Sad, Faculty of Technical Sciences, Department of Graphic Engineering and Design. This article is an open access article distributed under the terms and conditions of the Creative Commons Attribution license 3.0 Serbia

(http://creativecommons.org/licenses/by/3.0/rs/). 